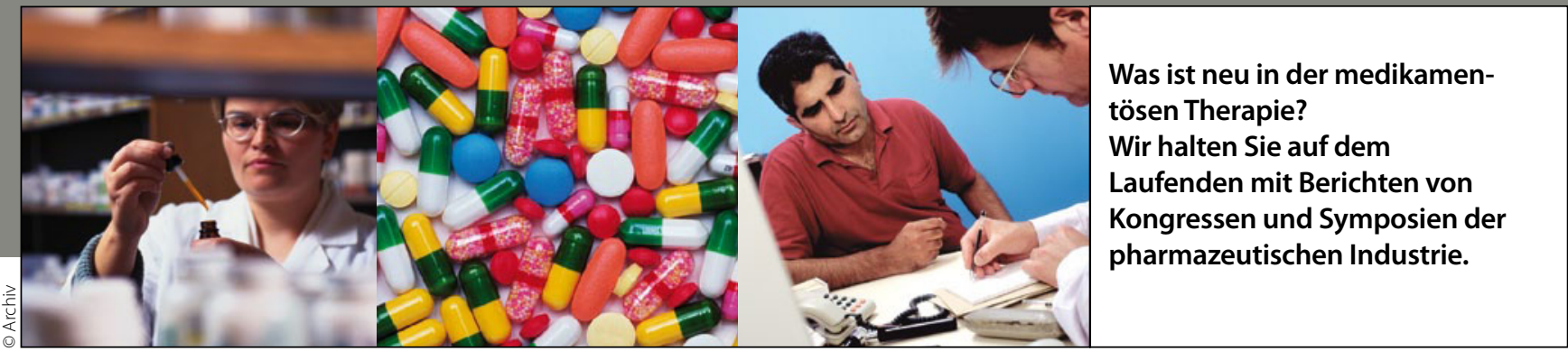

Typ-2-Diabetes

\title{
Insulindetemir trickst Hypoglykämien aus
}

— Die neue Leitlinie der Deutschen Diabetes-Gesellschaft (DDG) empfiehlt den Einstieg in die Insulintherapie, wenn mit der Kombination von zwei oralen Antidiabetika der $\mathrm{HbA}_{1 \mathrm{c}}$ über drei bis sechs Monate nicht $<6,5 \%$ bzw. dem individuellen $\mathrm{HbA}_{1 \mathrm{c}}$-Zielwert gehalten werden kann. „Bei diesen Patienten besteht meist eine Nüchternhyperglykämie", sagte Dr. Marcel Kaiser, Frankfurt/M. Deshalb ist es sinnvoll, die Insulintherapie mit einem Basalinsulin zu starten und zwar in Kombination mit der bisherigen oralen Therapie. „Sinn einer solchen Basalinsulintherapie ist es, den Nüchternblutzucker zu senken und somit für den Diabetiker optimale Bedingungen für den Start in den Tag zu schaffen", so Kaiser.

\section{In der ersten Nachthälfte reagiert der} Körper am empfindlichsten auf Insulin Für die basale Insulintherapie stehen neben modernen lang wirksamen Insulinanaloga wie Insulindetemir (Levemir ${ }^{\circledast}$ ) auch $\mathrm{NPH}$-Insuline zur Verfügung. Bei einer abendlichen Gabe eines NPH-Insulins ergeben sich jedoch zwei Problembereiche. Es kommt zu einer starken Anflutung des Insulins in der ersten Nachthälfte, in einer Zeit, wo der Körper auf Insulin am empfindlichsten reagiert. Daraus resultiert das erhöhte Hypoglykämierisiko. Im weiteren Verlauf nimmt die Wirkung des NPH-Insulins deutlich ab, sodass in den Morgenstunden weiterhin eine Hyperglykämie besteht. „Wird dann die NPH-Insulin-Dosis erhöht, steigt das Hypoglykämierisiko weiter an", so Kaiser.

Im Vergleich zu einem NPH-Insulin bietet Insulindetemir wesentliche Vorteile. Angesichts seiner Pharmakokinetik wirkt es in der ersten Nachthälfte schwächer und wegen seiner längeren Wirkdauer steht in den Morgenstunden mehr Insulin zur Verfügung. Dass durch die Gabe von Insulindetemir das Hypoglykämierisiko im Vergleich zu einem NPH-Insulin wesentlich gesenkt werden kann, wurde in entsprechenden Studien dokumentiert. Die Gesamtrate an Hypoglykämien nahm um 53\% und an nächtlichen Hypoglykämien sogar um $65 \%$ ab. „Diese günstigere Wirkung des Insulinanalogons spiegelt sich jedoch nicht in dem $\mathrm{HbA}_{1 \mathrm{c}}$-Wert wieder", so Kaiser. Vorteilhaft bei Insulindetemir ist auch, dass es den Gewichtsverlauf günstiger beeinflusst als andere lang wirksame Insulinanaloga.
Leichte nächtliche Hypoglykämien führen zu einer Beeinträchtigung der Leistungsfähigkeit mit entsprechenden Ausfällen am Arbeitsplatz. „Hypoglykämien sind auch von enormer sozioökonomischer Bedeutung", so Kaiser. Es sei deshalb sinnvoll, den primären Einsatz eines modernen Basalinsulins wie Insulindetemir zu empfehlen. Da mit fast allen Krankenkassen entsprechende Rabattverträge abgeschlossen werden konnten, sind Insulindetemir und $\mathrm{NPH}-$ Insuline kostengleich.

\section{- Dr. med. Peter Stiefelhagen}

Quelle: Pressekonferenz auf dem 117. Internistenkongress, Wiesbaden, Mai 2011 (Veranstalter: Novo Nordisk)

\section{Infliximab bei Morbus Crohn}

\section{Nicht mehr nur für schwere Fälle zugelassen}

— Die Europäische Kommission hat für Infliximab (Remicade ${ }^{\circledast}$ ) Ende April die Zulassung erweitert: Der TNF- $\alpha$-Antikörper ist nun auch zur Behandlung von mäßiggradigem aktivem Morbus Crohn zugelassen (bisher für schwergradigen aktiven Morbus Crohn).

Behandelt werden können jetzt auch Patienten mit mäßiggradigen Erkrankungen, die trotz eines vollständigen und adäquaten Therapieregimes mit einem Kortikosteroid und/oder einem Immunsuppressivum nicht auf die Behandlung ansprachen, oder bei denen eine Unverträglichkeit bzw. Gegenanzeige für diese Therapien vorliegt. Die Europäische Kom- mission erteilte die Zulassung auf der Grundlage von Daten aus mehreren relevanten Studien zu Infliximab einschließlich der SONIC-Studie (Study of Biologic and Immunomodulator Naive Patients in Crohn's Disease). In Letzterer wurde Infliximab mit Azathioprin bei Patienten mit mäBig- bis schwergradigem Morbus Crohn verglichen. Die klinische Prüfung zeigte, dass ein signifikant größerer Anteil von Patienten, die Infliximab in Kombination mit Azathioprin oder nur Infliximab erhielten, im Vergleich zu Patienten unter Azathioprin eine steroidfreie Remission und vollständige Mukosaheilung - wichtige Therapieziele bei Morbus Crohn - erreichte. 